\title{
Efficient electromagnetic boundary conditions to accelerate optimization of RF devices
}

\author{
Yong-Heui Cho \\ School of Information and Communication Engineering \\ Mokwon University, Daejeon, 302-729, Republic of Korea
}

\begin{abstract}
To achieve efficient field formulations and fast numerical computations, the reciprocal relations and equivalence between tangential and normal boundary conditions for electromagnetic fields are discussed in terms of the Maxwell's differential equations. Using the equivalence of each boundary condition, we propose the six essential boundary conditions, which may be applicable to matching electromagnetic discontinuities to efficiently design RF devices. In order to verify our approach, the reflection characteristics of a rectangular waveguide step are compared with respect to six essential boundary conditions.
\end{abstract}

Keywords: Wireless Communication System, RF Device, Electromagnetic Wave, Boundary Condition.

\section{INTRODUCTION}

In a wireless communication system [1]-[3], the performance of RF devices is of utmost importance to achieve high SNR (Signal to Noise Ratio) and reliable communication link. For better performance, a RF device should have low reflection coefficient, low insertion loss, simple structure, easy fabrication, etc. Since many researchers have investigated the $\mathrm{RF}$ scattering mechanism, the RF design procedures are well established.

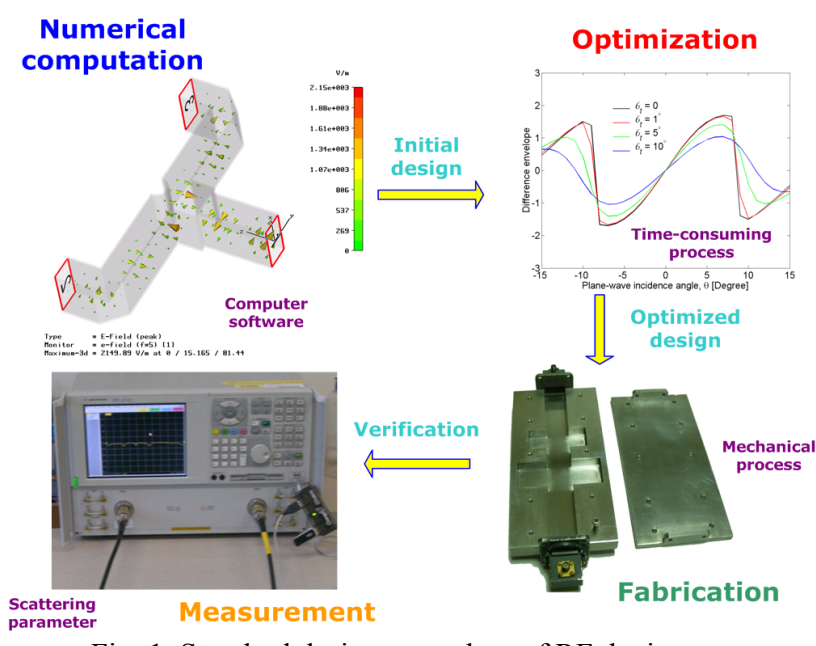

Fig. 1. Standard design procedure of RF devices

Fig. 1 illustrates the standard design procedure for RF devices which is composed of numerical computation,

\footnotetext{
${ }^{*}$ Corresponding author.E-mail : yhcho@mokwon.ac.kr Manuscript received Oct 10, 2011 ; accepted Oct.28, 2011
}

optimization [4], fabrication, and measurement. Among these steps, the optimization [4] is very time-consuming and deeply related to numerical computation scheme. However, when we have fast numerical computation algorithm, we can efficiently accelerate the optimization step. Numerical computation algorithms for RF devices include mode-matching technique, MoM (Method of Moments), FDM (Finite Difference Method), FEM (Finite Element Method), etc. For canonical structures such as waveguide structures, microwave resonators, and standard scatterers, the mode-matching technique is well known for fast computation time and low memory consumption. To apply the mode-matching technique, the electromagnetic boundary conditions (BCs) should be invoked beforehand. The enforcement of electromagnetic BCs is also one of fundamental problems in the application of a classical electromagnetics [5][12]. In [5]-[8], the concept of BCs has been extensively investigated and extended by means of four vector potentials, generalized impedance BCs related to higher order derivatives, differential forms, and the uniqueness theorem, respectively. The dependent relations of tangential and normal BCs are discussed and proved with the Stokes' theorem [9]. In [11], [12], the normal BCs for a planar surface are proposed based on the Maxwell's equations.

In this work, we present a simple yet rigorous proof of the reciprocal relations and equivalence between tangential and normal BCs in terms of the Maxwell's differential equations, thus facilitating more rapid and efficient numerical computations. Utilizing this equivalency, we will present six essential BCs. Numerical computations will be also performed to check the validity of applying the essential BCs. In Sect. 4, we will prove that the $\mathrm{BCs}$ composed of normal fields produce

This study was financially supported by academic research fund of Mokwon University in 2011. 
simpler matrix equations than those of tangential fields.

\section{RELATIONSHIP BETWEEN TANGENTIAL AND NORMAL BOUNDARY CONDITIONS}

Let's consider the sourceless Maxwell's equations as

$$
\begin{aligned}
& \bar{\nabla} \times \bar{E}=i \omega \bar{B} \\
& \bar{\nabla} \times \bar{H}=-i \omega \bar{D} \\
& \bar{\nabla} \cdot \bar{D}=0 \\
& \bar{\nabla} \cdot \bar{B}=0
\end{aligned}
$$

where we use the $e^{-i \omega t}$ time convention. In terms of tangential fields $\bar{E}_{t}, \bar{H}_{t}$ and normal fields $\bar{D}_{n}, \bar{B}_{n}$ with respect to an electromagnetic boundary layer in Fig. 2, the Maxwell's equations from (1) to (4) can be simplified as

$$
\begin{aligned}
& \bar{\nabla}_{t} \times \bar{E}_{t}=i \omega \bar{B}_{n} \\
& \bar{\nabla}_{t} \times \bar{H}_{t}=-i \omega \bar{D}_{n} \\
& \bar{\nabla}_{t} \cdot \bar{E}_{t}=-\partial E_{n} / \partial n+\rho_{e} / \varepsilon \\
& \bar{\nabla}_{t} \cdot \bar{H}_{t}=-\partial H_{n} / \partial n+\rho_{m} / \mu
\end{aligned}
$$

where $\rho_{e}, \rho_{m}$ are equivalent electric and magnetic charge densities, respectively, which are frequently introduced in waveguide discontinuity problems. The concept of equivalent charge densities will be explained in Sect. 3 .

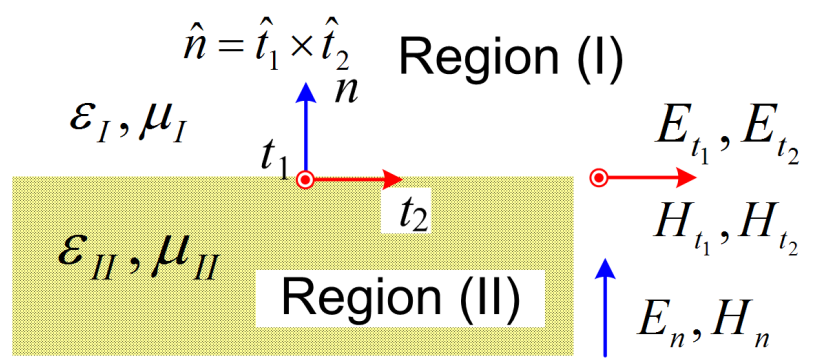

Fig. 2. Geometry of an electromagnetic boundary layer

For the tangential fields $\bar{E}_{t}, \bar{H}_{t}$ in a sourceless medium, using (1) and (2), the ordinary matching BCs are given by [13]

$$
\bar{E}_{t}^{I}=\bar{E}_{t}^{I I} \text { and } \bar{H}_{t}^{I}=\bar{H}_{t}^{I I} .
$$

Subtracting (5) and (6) for regions (I) and (II) gives the relations between tangential and normal fields as

$$
\frac{\partial}{\partial t_{1}}\left(E_{t_{2}}^{I}-E_{t_{2}}^{I I}\right)-\frac{\partial}{\partial t_{2}}\left(E_{t_{1}}^{I}-E_{t_{1}}^{I I}\right)=i \omega\left(B_{n}^{I}-B_{n}^{I I}\right)
$$

$\frac{\partial}{\partial t_{1}}\left(H_{t_{2}}^{I}-H_{t_{2}}^{I I}\right)-\frac{\partial}{\partial t_{2}}\left(H_{t_{1}}^{I}-H_{t_{1}}^{I I}\right)=-i \omega\left(D_{n}^{I}-D_{n}^{I I}\right),(11)$

where $\hat{t}_{1} \perp \hat{t}_{2} \perp \hat{n}, \hat{t}_{1} \times \hat{t}_{2}=\hat{n}$, and $\hat{t}_{1,2}$ and $\hat{n}$ are the tangential and normal unit vectors to a boundary layer in Fig. 2, respectively. Inserting (9) into (10) and (11), we obtain the auxiliary BCs as [9]

$$
B_{n}^{I}=B_{n}^{I I} \text { and } D_{n}^{I}=D_{n}^{I I}
$$

Even though the normal BCs (12) can be alternatively derived with a conceptual infinitesimal cylinder [13], (9) and (12) show that tangential BCs result in normal BCs, thus confirming that tangential and normal $\mathrm{BCs}$ are not independent each other.

Similarly, manipulating (7), (8), and (9), we also get the another auxiliary $\mathrm{BCs}$ as

$$
\begin{aligned}
& \frac{\partial}{\partial n} E_{n}^{I}-\frac{\rho_{e}^{I}}{\varepsilon_{I}}=\frac{\partial}{\partial n} E_{n}^{I I}-\frac{\rho_{e}^{I I}}{\varepsilon_{I I}} \\
& \frac{\partial}{\partial n} H_{n}^{I}-\frac{\rho_{m}^{I}}{\mu_{I}}=\frac{\partial}{\partial n} H_{n}^{I I}-\frac{\rho_{m}^{I I}}{\mu_{I I}} .
\end{aligned}
$$

In view of the Maxwell's divergence equations, (3) and (4), and the uniqueness theorem in electromagnetics [13], the normal BCs (12) should be equivalent to (13) and (14) when tangential BCs (9) are given.

For the normal fields in a sourceless medium, we can select the matching $\mathrm{BCs}$ as

$$
\begin{aligned}
& D_{n}^{I}=D_{n}^{I I} \text { and } B_{n}^{I}=B_{n}^{I I} \\
& E_{t_{1}}^{I}=E_{t_{1}}^{I I} \text { and } H_{t_{1}}^{I}=H_{t_{1}}^{I I} .
\end{aligned}
$$

It should be noted that we can replace (16) for the $t_{1}$-axis with that for the $t_{2}$-axis as

$$
E_{t_{2}}^{I}=E_{t_{2}}^{I I} \text { and } H_{t_{2}}^{I}=H_{t_{2}}^{I I}
$$

Applying (15) and (16) to (10) and (11), we may obtain the additional BCs as

$$
\begin{aligned}
& \frac{\partial}{\partial t_{1}}\left(E_{t_{2}}^{I}-E_{t_{2}}^{I I}\right)=0 \\
& \frac{\partial}{\partial t_{1}}\left(H_{t_{2}}^{I}-H_{t_{2}}^{I I}\right)=0 .
\end{aligned}
$$

Based on (18) and (19), we define the tangential field relations for region (I) and (II) as 


$$
\begin{aligned}
& E_{t_{2}}^{I}=E_{t_{2}}^{I I}+C_{E}\left(t_{2}, n\right) \\
& H_{t_{2}}^{I}=H_{t_{2}}^{I I}+C_{H}\left(t_{2}, n\right),
\end{aligned}
$$

where $C_{E}\left(t_{2}, n\right)$ and $C_{H}\left(t_{2}, n\right)$ are arbitrary functions of only one-variable $n$. Inserting (20) and (21) into (1) and (2) yields

$$
\begin{aligned}
& \bar{\nabla} \times\left[C_{E}\left(t_{2}, n\right) \hat{t}_{2}\right]=i \omega \mu_{I I} C_{H}\left(t_{2}, n\right) \hat{t}_{2} \\
& \bar{\nabla} \times\left[C_{H}\left(t_{2}, n\right) \hat{t}_{2}\right]=-i \omega \varepsilon_{I I} C_{E}\left(t_{2}, n\right) \hat{t}_{2}
\end{aligned}
$$

In order to simultaneously satisfy (22) and (23), we should have $C_{E}\left(t_{2}, n\right)=C_{H}\left(t_{2}, n\right)=0$. This means that (15) and (16) yield (17) and in the same manner (15) and (17) result in (16).

\section{ESSENTIAL BOUNDARY CONDITIONS AND THEIR EQUIVALENCY}

Utilizing the reciprocal relationship between tangential and normal BCs in Sect. 2, we can define the essential BCs which are equivalent each other. Since the tangential BCs (9) produce the normal BCs (12), (13), and (14) and vice versa, we obtain

\begin{tabular}{|c|c|c|}
\hline Condition & Subcondition (a) & Subcondition (b) \\
\hline (I) & $E_{t_{1}}$ and $H_{t_{1}}$ & $E_{t_{2}}$ and $H_{t_{2}}$ \\
\hline (II) & $\varepsilon E_{n}$ and $\mu H_{n}$ & $\begin{array}{l}E_{t_{1}} \text { and } H_{t_{1}} \\
\text { or } E_{t_{2}} \text { and } H_{t_{2}}\end{array}$ \\
\hline (III) & $\begin{array}{l}\frac{\partial E_{n}}{\partial n}-\frac{\rho_{e}}{\varepsilon} \text { and } \\
\frac{\partial H_{n}}{\partial n}-\frac{\rho_{m}}{\mu}\end{array}$ & $\begin{array}{l}E_{t_{1}} \text { and } H_{t_{1}} \\
\text { or } E_{t_{2}} \text { and } H_{t_{2}}\end{array}$ \\
\hline (IV) & $\begin{array}{c}\varepsilon E_{n} \text { and } \\
\frac{\partial H_{n}}{\partial n}-\frac{\rho_{m}}{\mu}\end{array}$ & $E_{t_{1}}$ and $E_{t_{2}}$ \\
\hline (V) & $\begin{array}{c}\frac{\partial E_{n}}{\partial n}-\frac{\rho_{e}}{\varepsilon} \text { and } \\
\mu H_{n}\end{array}$ & $H_{t_{1}}$ and $H_{t_{2}}$ \\
\hline (VI) & $\begin{array}{c}\frac{\partial E_{n}}{\partial n}-\frac{\rho_{e}}{\varepsilon} \text { and } \\
\mu H_{n}\end{array}$ & $\begin{array}{c}\varepsilon E_{n} \text { and } \\
\frac{\partial H_{n}}{\partial n}-\frac{\rho_{m}}{\mu}\end{array}$ \\
\hline
\end{tabular}
the six equivalent BCs, any one of which makes us have unique solutions.

Table 1. Six essential boundary conditions
Table 1 shows the six essential BCs for electromagnetic fields, where each essential BC is composed of Subconditions (a) and (b) in Table 1. To understand Table 1, let's compare and get equivalency of the tangential and normal BCs. Continuity of tangential electric fields (9) is equivalent to that of normal magnetic flux densities (12) and normal derivative of normal electric fields (13). Similarly, continuity of tangential magnetic fields (9) does the same to that of normal electric flux densities (12) and normal derivative of normal magnetic fields (14). In addition, these continuities are vice versa. This indicates that the tangential and normal BCs in (9), (12), (13), and (14) have equivalent relations which are overtly organized in Table 1.

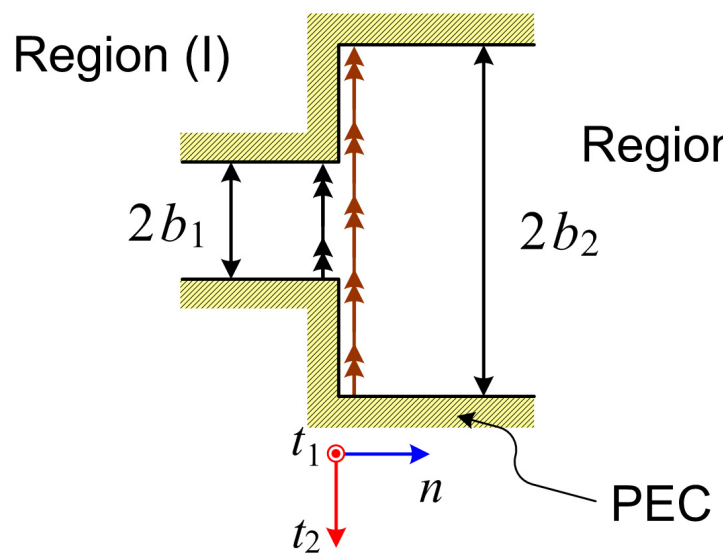

Fig. 3. Geometry of a PEC abrupt step

In the derivation of tangential and normal BCs, we assume there may be equivalent charge densities, $\rho_{e}$ and $\rho_{m}$, in geometrical discontinuity. To figure out this assumption, let's regard a PEC (Perfect Electric Conductor) abrupt step in Fig. 3. For the Conditions (III) through (VI), we define the equivalent electric and magnetic surface charge densities $\rho_{e s}$ and $\rho_{m s}$ at the waveguide step in Fig. 3. As such, we can get

$$
\begin{aligned}
& \rho_{e s}^{I}=-\varepsilon_{1} \bar{E}_{t}^{I} \cdot \hat{n}_{I} \text { and } \rho_{e s}^{I I}=0 \\
& \rho_{m s}^{I}=0 \text { and } \rho_{m s}^{I I}=-\mu_{2} \bar{H}_{t}^{I I} \cdot \hat{n}_{I I}
\end{aligned}
$$

where $\hat{n}_{I}$ and $\hat{n}_{I I}$ are the outward normal vectors to the waveguides in regions (I) and (II), respectively. The definition in (24) and (25) can be understood in terms of electromagnetic BCs. As shown in Fig. 3, the tangential electric field $\bar{E}_{t}$ is normal to the PEC surface. This means that there should be surface electric charge density $\rho_{e s}$ on the PEC surface [13]. When we set up the simultaneous equations for Fig. 3 , the integration interval for the electric field is from $-b_{2}$ to $b_{2}$. Thus, $\rho_{e s}$ is nonzero for region (I) and zero for region (II). This is because the edge points of region (I) at $t_{2}= \pm b_{1}$ are within the integration interval $\left(-b_{2}, b_{2}\right)$. The formula (25) 
can also be figured out in terms of magnetic $\mathrm{BC}$ in which the normal magnetic field toward a PEC surface should be zero. The integration interval for the tangential magnetic field $\bar{H}_{t}$ is defined from $-b_{1}$ to $b_{1}$. The magnetic $\mathrm{BC}$ is satisfied for region (I), whereas that does not for region (II) due to truncation of the integral interval $\left(-b_{1}, b_{1}\right)$. As a result, we have to introduce $\rho_{m s}$ for region (II)

\section{NUMERICAL ANALYSIS}

In order to verify the equivalency of matching BCs in Table 1, we consider a rectangular waveguide step shown in Fig. 4.

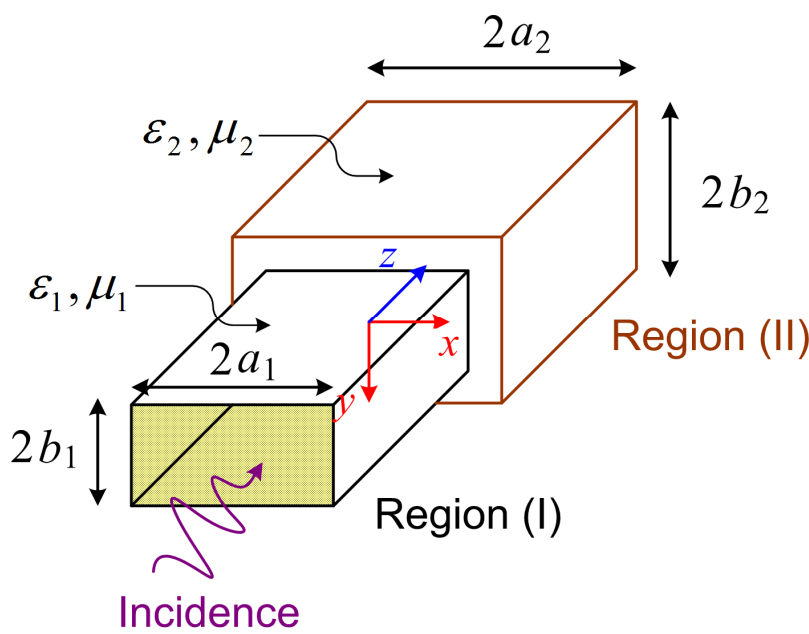

Fig. 4. Geometry of a rectangular waveguide step

Assume that an incident $\mathrm{TE}_{m_{s} n_{s}}$ mode impinges upon a step. A standard mode-matching technique will be applied and the number of modes for regions (I) and (II) will be chosen to facilitate the mode selection criterion in [14]. The incident and reflected $H_{z}$ fields in region(I) $(z \leq 0)$ are

$$
\begin{aligned}
& H_{z}^{i}(\bar{r})=\cos a_{m_{s}}^{I}\left(x+a_{1}\right) \cos b_{n_{s}}^{I}\left(y+b_{1}\right) e^{i \beta_{m_{s} n_{s}}^{I}{ }^{z}} \\
& H_{z}^{r}(\bar{r})=-\cos a_{m_{s}}^{I}\left(x+a_{1}\right) \cos b_{n_{s}}^{I}\left(y+b_{1}\right) e^{-i \beta_{m_{s} n_{s}}^{I}{ }^{z}},
\end{aligned}
$$

where $\quad a_{m}^{I}=m \pi / 2 a_{1} \quad, \quad b_{n}^{I}=n \pi / 2 b_{1}$, $k_{1}^{2}=\left(k_{m n}^{I}\right)^{2}+\left(\beta_{m n}^{I}\right)^{2}$, and $\left(k_{m n}^{I}\right)^{2}=\left(a_{m}^{I}\right)^{2}+\left(b_{n}^{I}\right)^{2}$. In region $(\mathrm{I})$, scattered electromagnetic fields are represented as

$$
\begin{aligned}
& E_{z}^{I}(\bar{r})=\sum_{m=0}^{\infty} \sum_{n=0}^{\infty} p_{m n}^{I} \sin a_{m}^{I}\left(x+a_{1}\right) \sin b_{n}^{I}\left(y+b_{1}\right) e^{-i \beta_{m n}^{I} z} \\
& H_{z}^{I}(\bar{r})=\sum_{m=0}^{\infty} \sum_{n=0}^{\infty} q_{m n}^{I} \cos a_{m}^{I}\left(x+a_{1}\right) \cos b_{n}^{I}\left(y+b_{1}\right) e^{-i \beta_{m n}^{I} z}
\end{aligned}
$$

where $m+n \neq 0$. In region (II) $(z>0)$, transmitted fields are

$$
\begin{aligned}
& E_{z}^{I I}(\bar{r})=\sum_{m=0}^{\infty} \sum_{n=0}^{\infty} p_{m n}^{I I} \sin a_{m}^{I I}\left(x+a_{2}\right) \sin b_{n}^{I I}\left(y+b_{2}\right) e^{i \beta_{m n}^{I I} z} \\
& H_{z}^{I I}(\bar{r})=\sum_{m=0}^{\infty} \sum_{n=0}^{\infty} q_{m n}^{I I} \cos a_{m}^{I I}\left(x+a_{2}\right) \cos b_{n}^{I I}\left(y+b_{2}\right) e^{i \beta_{m n}^{I I} z},
\end{aligned}
$$

where $\quad a_{m}^{I I}=m \pi / 2 a_{2} \quad, \quad b_{n}^{I I}=n \pi / 2 b_{2}$ $k_{2}^{2}=\left(k_{m n}^{I I}\right)^{2}+\left(\beta_{m n}^{I I}\right)^{2}$, and $\left(k_{m n}^{I I}\right)^{2}=\left(a_{m}^{I I}\right)^{2}+\left(b_{n}^{I I}\right)^{2}$.

Multiplying the $E_{x}$ continuity at $z=0$ by $\cos a_{l}^{I I}\left(x+a_{2}\right) \sin b_{k}^{I I}\left(y+b_{2}\right) \quad$ and integrating over $-a_{2}<x<a_{2}$ and $-b_{2}<y<b_{2}$ results in

$$
\begin{aligned}
& \sum_{m, n}\left[\left(Y_{m n}^{I} a_{m}^{I} p_{m n}^{I}+b_{n}^{I} q_{m n}^{I}\right) A_{m n}^{I} G_{m l}(a) F_{n k}(b)\right. \\
+ & \left.\left(Y_{m n}^{I I} a_{m}^{I I} p_{m n}^{I I}-b_{n}^{I I} q_{m n}^{I I}\right) A_{m n}^{I I} a_{2} b_{2} \alpha_{m} \delta_{m l}^{n k}\right]=0,
\end{aligned}
$$

where $\delta_{m l}^{n k}=\delta_{m l} \delta_{n k}$,

$$
\alpha_{m}=\delta_{m 0}+1= \begin{cases}2 & (m=0) \\ 1 & (m=1,2, \cdots)\end{cases}
$$

$G_{m l}(a)=\frac{2 a_{l}^{I I}}{\left(a_{l}^{I I}\right)^{2}-\left(a_{m}^{I}\right)^{2}} \cos \left(a_{l}^{I I} a_{2}-a_{m}^{I} a_{1}\right) \sin \left(a_{l}^{I I}-a_{m}^{I}\right) a_{1}$

$F_{n k}(b)=\frac{2 b_{n}^{I}}{\left(b_{k}^{I I}\right)^{2}-\left(b_{n}^{I}\right)^{2}} \cos \left(b_{k}^{I I} b_{2}-b_{n}^{I} b_{1}\right) \sin \left(b_{k}^{I I}-b_{n}^{I}\right) b_{1}$.

By enforcing the $E_{y}$ continuity at $z=0$ and multiplying it by $\sin a_{l}^{I I}\left(x+a_{2}\right) \cos b_{k}^{I I}\left(y+b_{2}\right)$, we obtain

$$
\begin{aligned}
& \sum_{m, n}\left[\left(Y_{m n}^{I} b_{n}^{I} p_{m n}^{I}-a_{m}^{I} q_{m n}^{I}\right) A_{m n}^{I} F_{m l}(a) G_{n k}(b)\right. \\
+ & \left.\left(Y_{m n}^{I I} b_{n}^{I I} p_{m n}^{I I}+a_{m}^{I I} q_{m n}^{I I}\right) A_{m n}^{I I} a_{2} b_{2} \alpha_{n} \delta_{m l}^{n k}\right]=0 .
\end{aligned}
$$

Similarly, from the $H_{x}$ and $H_{y}$ continuities at $z=0$, we get, respectively,

$$
\begin{aligned}
& \sum_{m, n}\left[\left(b_{n}^{I} p_{m n}^{I}-Z_{m n}^{I} a_{m}^{I} q_{m n}^{I}\right) B_{m n}^{I} a_{1} b_{1} \alpha_{n} \delta_{m l}^{n k}\right. \\
& \left.-\left(b_{n}^{I I} p_{m n}^{I I}+Z_{m n}^{I I} a_{m}^{I I} q_{m n}^{I I}\right) B_{m n}^{I I} F_{l m}(a) G_{k n}(b)\right] \\
& =-2 Z_{m_{s} n_{s}}^{I} a_{m_{s}}^{I} B_{m_{s} n_{s}}^{I} a_{1} b_{1} \alpha_{n_{s}} \delta_{m_{s} l}^{n_{s} k}
\end{aligned}
$$




$$
\begin{aligned}
& \sum_{m, n}\left[\left(a_{m}^{I} p_{m n}^{I}+Z_{m n}^{I} b_{n}^{I} q_{m n}^{I}\right) B_{m n}^{I} a_{1} b_{1} \alpha_{m} \delta_{m l}^{n k}\right. \\
& \left.-\left(a_{m}^{I I} p_{m n}^{I I}-Z_{m n}^{I I} b_{n}^{I I} q_{m n}^{I I}\right) B_{m n}^{I I} G_{l m}(a) F_{k n}(b)\right] \\
& =2 Z_{m_{s} n_{s}}^{I} b_{n_{s}}^{I} B_{m_{s} n_{s}}^{I} a_{1} b_{1} \alpha_{m_{s}} \delta_{m_{s} n_{s} k}^{n_{s}} .
\end{aligned}
$$

In terms of normal fields, we enforce the $\varepsilon E_{z}$ and $\mu H_{z}$ continuities at $z=0$ to obtain the scattering relations as, respectively,

$$
\begin{aligned}
& \sum_{m, n}\left[p_{m n}^{I} a_{1} b_{1} \delta_{m l}^{n k}-\frac{\varepsilon_{2}}{\varepsilon_{1}} p_{m n}^{I I} F_{l m}(a) F_{k n}(b)\right]=0 \\
& \sum_{m, n}\left[q_{m n}^{I} G_{m l}(a) G_{n k}(b)-\frac{\mu_{2}}{\mu_{1}} q_{m n}^{I I} a_{2} b_{2} \alpha_{m} \alpha_{n} \delta_{m l}^{n k}\right]=0
\end{aligned}
$$

Similarly, applying the $\partial E_{z} / \partial z-\rho_{e} / \varepsilon$ and $\partial H_{z} / \partial z-\rho_{m} / \mu$ continuities at $z=0$ yields, respectively,

$$
\begin{aligned}
& \sum_{m, n}\left\{\left[Y_{m n}^{I}\left(k_{l k}^{I I}\right)^{2} p_{m n}^{I}+C_{m l}^{n k} q_{m n}^{I}\right] A_{m n}^{I} F_{m l}(a) F_{n k}(b)\right. \\
& \left.+p_{m n}^{I I} \beta_{m n}^{I I} a_{2} b_{2} \delta_{m l}^{n k}\right\}=0 \\
& \sum_{m, n}\left\{q_{m n}^{I} \beta_{m n}^{I} a_{1} b_{1} \alpha_{m} \alpha_{n} \delta_{m l}^{n k}\right. \\
& \left.+\left[D_{m l}^{n k} p_{m n}^{I I}+Z_{m n}^{I I}\left(k_{l k}^{I}\right)^{2} q_{m n}^{I I}\right] B_{m n}^{I I} G_{l m}(a) G_{k n}(b)\right\} \\
& =2 \beta_{m_{s} n_{s}}^{I} a_{1} b_{1} \alpha_{m_{s}} \alpha_{n_{s}} \delta_{m_{s} l}^{n_{s} k},
\end{aligned}
$$

where

$$
\begin{aligned}
& C_{m l}^{n k}=\frac{\left(a_{l}^{I I}\right)^{2}\left(b_{n}^{I}\right)^{2}-\left(a_{m}^{I}\right)^{2}\left(b_{k}^{I I}\right)^{2}}{a_{m}^{I} b_{n}^{I}} \\
& D_{m l}^{n k}=\frac{\left(a_{l}^{I}\right)^{2}\left(b_{n}^{I I}\right)^{2}-\left(a_{m}^{I I}\right)^{2}\left(b_{k}^{I}\right)^{2}}{a_{m}^{I I} b_{n}^{I I}} .
\end{aligned}
$$

Manipulating (32), (36) through (42), we can compose of the six essential BCs shown in Table 1 . When $\hat{t}_{1}=\hat{x}, \hat{t}_{2}=\hat{y}$, $\hat{n}=\hat{z}$, the essential BCs for the geometry in Fig. 4 are formulated in Table 2 .

Table 2. Essential boundary conditions for a waveguide step

\begin{tabular}{|c|c|c|}
\hline Condition & Subcondition (a) & Subcondition (b) \\
\hline (I) & (32) and (36) & $(37)$ and (38) \\
\hline (II) & (39) and (40) & $\begin{array}{l}(32) \text { or (36) } \\
(37) \text { or (38) }\end{array}$ \\
\hline (III) & (41) and (42) & $\begin{array}{l}(32) \text { or (36) } \\
(37) \text { or (38) }\end{array}$ \\
\hline (IV) & (39) and (42) & $(32)$ and (36) \\
\hline (V) & (41) and (40) & $(37)$ and (38) \\
\hline (VI) & (41) and (40) & $(39)$ and (42) \\
\hline
\end{tabular}

For instance, let's compare the simultaneous equations for Conditions (I) and (VI). The equation set for Condition (I) shown in Table 2 is obtained with the standard tangential field matching and that for Condition (VI) does with the normal field matching proposed in this work. Condition (I) results in much more complex equations than Condition (VI), thus verifying that Condition (VI) is numerically very efficient for most practical applications.

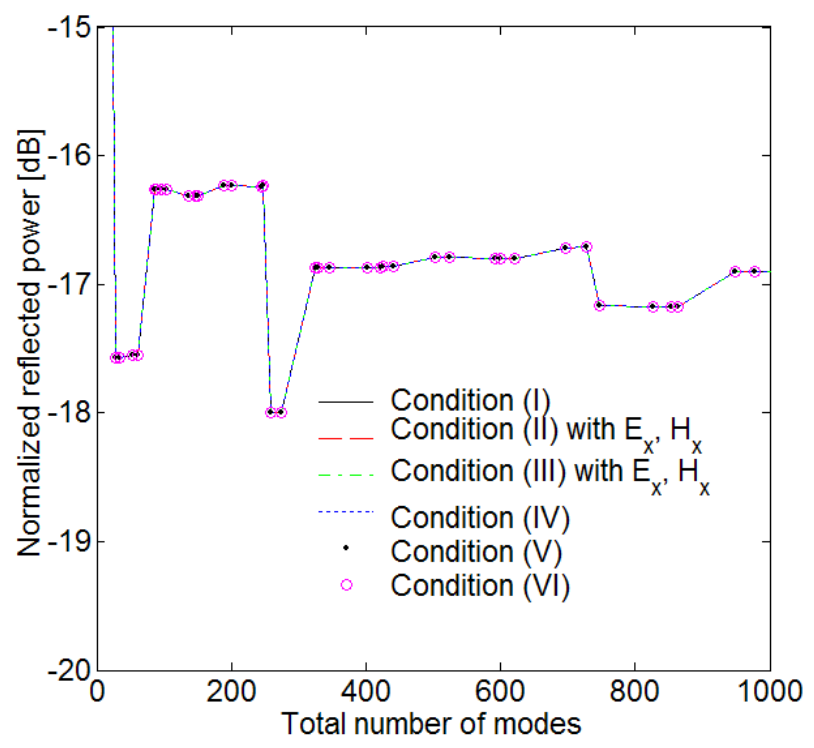

Fig. 5. Convergence characteristics of normalized reflected power with $f=5[\mathrm{GHz}], \varepsilon_{1}=\varepsilon_{0}, \mu_{1}=\mu_{0}, \varepsilon_{1}=2 \varepsilon_{0}$, $\mu_{1}=2 \mu_{0}, a_{1}=2 b_{1}=20.19[\mathrm{~mm}], a_{2}=2 b_{2}=54.61$ $[\mathrm{mm}]$

Fig. 5 shows the convergence characteristics of normalized reflected power versus the total number of modes with respect to the $\mathrm{TE}_{10}$ mode incidence. Numerical computations illustrate that the convergence characteristics of the BCs (I) through (VI) agree well with each other.

\section{CONCLUSION}

By studying the equivalency of tangential and normal electromagnetic boundary conditions, we propose the six essential boundary conditions. We showed that the six essential boundary conditions composed of tangential and normal fields are identical and interchangeable each other. For normal boundary conditions, a novel concept of equivalent charge density is important to obtain proper electromagnetic solutions. Numerical computations for a waveguide step show that our essential boundary conditions are almost identical each other. The boundary conditions composed of normal fields are more efficient than those for tangential fields in terms of computational complexity. In further work, we will investigate the generalization of boundary conditions for general media including metamaterial, plasma, and anisotropic material. 


\section{REFERENCES}

[1] S.-H. Lee, "A dynamic channel allocation algorithm based on time constraints in cellular mobile networks," International Journal of Contents, vol. 1, no. 2, Oct. 2005, pp. 31-34.

[2] I. Ituen and G.-H. Sohn, "The environmental applications of wireless sensor networks," International Journal of Contents, vol. 3, no. 4, Dec. 2007, pp. 1-7.

[3] S.-J. Kim, M.-S. Hwang, and Y.-M. Kim, "Implementation of low power algorithm for near distance wireless communication and RFID/USN systems," International Journal of Contents, vol. 7, no. 1, March 2011, pp. 1-7.

[4] J.-Y. Kim and D.-Y. Yang, "Genetic algorithm optimization of LNA for wireless applications in $2.4 \mathrm{GHz}$ band," International Journal of Contents, vol. 2, no. 1, March 2006, pp. 29-33.

[5] L. F. Jelsma, E. D. Tweed, R. L. Phillips, and R. W. Taylor, "Boundary conditions for the four vector potential," IEEE Trans. Microwave Theory Tech., vol. 18, no. 9, Sept. 1970, pp. 648-650.

[6] T. B. A. Senior and J. L. Volakis, "Derivation and application of a class of generalized boundary conditions," IEEE Trans. Antennas Propagat., vol. 37, no. 12, Dec. 1989, pp. 1566-1572.

[7] K. F. Warnick, R. H. Selfridge, and D. V. Arnold, "Electromagnetic boundary conditions and differential forms," IEE Proc. - Microw. Antennas Propag., vol. 142, no. 4, Aug. 1995, pp. 326-332.

[8] N. K. Nikolova, "Electromagnetic boundary conditions and uniqueness revisited," IEEE Antennas Propagat. Magazine, vol. 46, no. 5, Oct. 2004, pp. 141-149.

[9] C. Yeh, "Boundary conditions in electromagnetics," Phys. Rev. E, vol. 48, no. 2, Aug. 1993, pp. 1426-1427.

[10] I. V. Lindell and A. Sihvola, "Electromagnetic boundary condition and its realization with anisotropic metamaterial," Phys. Rev. E, vol. 79, no. 2, 2009.

[11] I. V. Lindell, H. Wallen, and A. Sihvola, "General electromagnetic boundary conditions involving normal field components," IEEE Antennas Wireless Propagat. Lett., vol. 8, no. 1, 2009, pp. 877-880.

[12] I. V. Lindell and A. H. Sihvola, "Electromagnetic boundary conditions defined in terms of normal field components," IEEE Trans. Antennas Propagat., vol. 58, no. 4, April 2010, pp. 1128-1135.

[13] C. A. Balanis, Advanced Engineering Electromagnetics, John Wiley \& Sons, 1989.

[14] R. Mittra, T. Itoh, and T.-S. Li, "Analytical and numerical studies of the relative convergence phenomenon arising in the solution of an integral equation by the moment method," IEEE Trans. Microwave Theory Tech., vol. 20, no. 2, Feb. 1972, pp. 96-104.

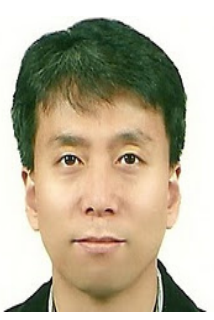

\section{Yong-Heui Cho}

$\mathrm{He}$ received the B. S. degree in Electronics Engineering from the Kyungpook National University, Daegu, Korea, in 1998, the M. S. and Ph. D. degrees in Electrical Engineering from the Korea Advanced Institute of Science and Technology (KAIST), Daejeon, Korea, in 2000 and 2002, respectively. From 2002 to 2003, he was a Senior Research Staff with the Electronics and Telecommunications Research Institute (ETRI), Daejeon, Korea. In 2003, he joined the School of Information and Communication Engineering, Mokwon University, Daejeon, Korea, where he is currently an Associate Professor. In 2011, he is on the sabbatical leave with the Department of Electrical and Computer Engineering, University of Massachusetts Amherst, MA, USA. His research interests include dispersion characteristics of waveguides, electromagnetic wave scattering, and design of reflectarrays. 